\title{
Oligodendroglioma-Like Component with Perinuclear Cytoplasmic Halos Present
}

National Cancer Institute

\section{Source}

National Cancer Institute. Oligodendroglioma-Like Component with Perinuclear

Cytoplasmic Halos Present. NCI Thesaurus. Code C96362.

A morphologic finding referring to the presence of a cellular infiltrate that is composed of cells with perinuclear cytoplasmic halos, resembling oligodendrocytes. 